\title{
Large Eddy Simulation of a Scale-model Turbofan for Fan Noise Source Diagnostic
}

\author{
C. Pérez Arroyo ${ }^{1}$, T. Leonard ${ }^{1}$, M. Sanjose ${ }^{1 *}$, S. Moreau' ${ }^{1}$, F. Duchaine ${ }^{2}$
}

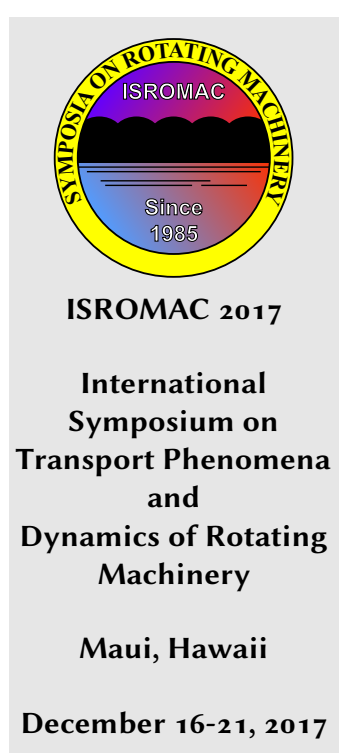

\begin{abstract}
\section{Keywords}

Fan stage - Large Eddy Simulation - aeroacoustic

${ }^{1}$ Department of Mechanical Engineering, University of Sherbrooke, Québec, Canada

${ }^{2}$ CFD Team and GlobC Team, CERFACS, Toulouse, France

*Corresponding author: Marlene.Sanjose@USherbrooke.ca
\end{abstract}

A wall-modeled Large Eddy Simulation (LES) of the turbulent flow in the NASA Source Diagnostic Test turbofan is successfully performed for the first time. A good agreement with aerodynamic measurements is observed for both Reynolds Averaged Navier-Stokes and LES results, although the LES provides better results in the tip regions where large coherent structures appear and no flow separation on the stator vanes is observed. In the LES the boundary layer naturally transition to turbulence on the blade suction side but remains quasi laminar over most of its pressure side. The rotor-wake turbulence yielding the stage broadband noise is then seen to be quasi isotropic. Transition on the downstream stator vanes is not triggered by the wake impingement but rather occurs at mid-chord. Finally, acoustics are investigated using both Ffowcs Williams \& Hawkings' and Goldstein's analogies from the recorded LES noise source on the stator vanes. The latter analogy provides levels much closer to the measurements especially at high frequencies, although the results are most likely still influenced by too coherent rotor tip secondary flow at low frequencies.

\section{INTRODUCTION}

Turbofan architecture has evolved toward an increasing contribution of the fan to the thrust. At approach conditions, the fan noise has become one of the main contributors with a main broadband content mostly in the forward arc of the engine. This will be all the more the case for future turboengines that will involve Ultra-High By-pass Ratio (UHBR) turbofans. For these architectures, fan noise is projected to become the dominant source overall. In a classical turbofan as the present configuration, the interaction between the wake of the rotor and equally-distributed Outlet-Guide Vanes (OGV) has been found to be the dominant mechanism that contributes to fan noise $[1,2]$. On the one hand, tonal noise can be more easily controlled by clever choice of blade counts according to Tyler \& Sofrin rules[3] and taking advantage of the duct filtering to have the first Blade Passing Frequency (BPF) cut-off for instance. Moreover proper nacelle and exhaust duct liners can also damp both forward and backward progagating tones [1]. On the other hand, broadband noise has a significant contribution much harder to control especially at high frequencies given the high number of cut-on modes for modern engines with low hub-to-tip ratio. Yet, with increasing noise regulations for commercial aircrafts, it becomes mandatory to get a better understanding of the noise generation and propagation for fan and OGV interaction broadband noise in order to be able to properly guide further noise reduction technology development.

Progress in High Performance Computing (HPC) has allowed for the correct prediction of complex flows around isolated parts of turbomachines with Reynolds Averaged Navier-Stokes (RANS), unsteady RANS, Large Eddy Simulations (LES) and high-fidelity Direct Numerical Simulations (DNS) as shown in reviews from Tucker [4] and McMullan et al. [5]. However, only the latter two methods (LES and DNS) can provide the multi-scale noise sources that yield broadband noise. A recent review from Gourdain et al. [6] gives an overview of LES industrial compressor flows and reports simulations with full stages highlighting the cost of LES for multistage compressors [7, 8]. But all these simulations involved low-pressure compressor with small blade spans, reduced rotor-stator distances and moderate Reynolds numbers based on the chord. Only de Laborderie et al. [7] considered the noise generation, but stressed the difficulties of predictions associated with limited passage simulations. More recently high-pressure turbines have been considered that involve both higher Mach and Reynolds numbers [9], and dealt with their noise generation and the propagation of combustion noise through them [10].

In that context, the "Fan Noise Source Diagnostic Test" (SDT) experimental set-up was investigated by NASA providing an extensive database of aerodynamic sources and aeroacoustic diagnostics of the broadband fan/OGV interaction mechanism $[11,12,13]$. This comprehensive study is dedicated to the validation of numerical and analytical methods and has recently become an AIAA benchmark case for fan broadband noise. The present work focuses on the only Large-Eddy Simulation (LES) of this configuration at the moment, in combination with acoustic analogies for noise 
prediction to investigate the rotor/stator interaction mechanism. This is the continuation of the preliminary work presented by Leonard et al. with converged flow and acoustic statistics [14]. In the latter, the streamwise turbulent length scale was extracted from the LES results and used in Hanson's model to predict the far-field noise. This study provides an in-depth analysis of the instantaneous aerodynamic features of this turbofan configuration and the comparison of two acoustic analogies for noise propagation. The prime focus has been on the noise generated by the fan/OGV pressure fluctuations and its link with the impingement of the passing turbulent rotor wakes. A precise description of the state of turbulence reaching the stator vanes leading edge is therefore mandatory, which strongly justified the LES. The NASA experiment is summarized in the next section. The complete numerical model and parameters are then provided. Finally both aerodynamic and acoustic results from the LES are shown.

\section{EXPERIMENTAL SET-UP}

As part of the NASA advanced Subsonic Technology Noise Reduction Program, several experiments $[11,13,15,12,16]$ have been carried out on the Source Diagnostic Test rig presented in Fig. 1(a) involving different geometries and counts of the rotor blades and stator outlet vanes [15]. The configuration studied here is the baseline configuration designed by General Electric Aircraft Engines which is composed of 22 rotor blades $\left(\mathrm{R}_{4}\right)$ and 54 radial stator vanes (Fig. 1(b)). This $1 / 5$ th scale model fan stage is representative of the secondary stream of a modern High Bypass-Ratio turbofan engine. Note that the experimental set-up does not involve a core flow.

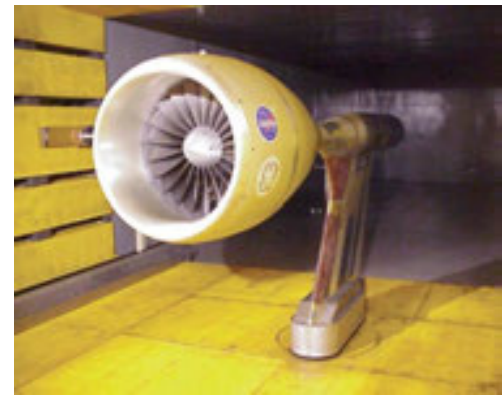

(a) turbofan with nacelle

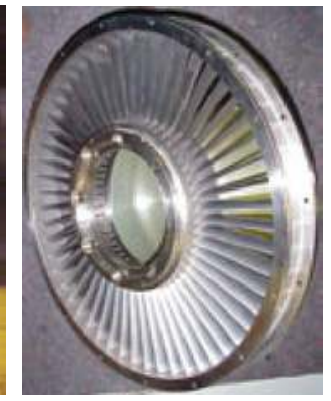

(b) baseline 54 radial guide vanes stator
Figure 1. Source Diagnostic Test test rig at NASA Glenn Low Speed Wind Tunnel.

The fan stage has been tested in take-off and approach conditions, but only the approach condition that has the most extensive experimental data set has been simulated numerically. Free-stream Mach number of 0.10 is imposed, to achieve acoustic flight effect and to provide far-field acoustic data representative of real take-off and approach conditions [13]. The rotational speed is $7808 \mathrm{rpm}$ corresponding to $61.7 \%$ of the rotor design speed.
The overall test program had many phases, including detailed flow field diagnostic measurements using laser Doppler and hot-wire anemometry $[12,16]$ and acoustic power measurements [13].

\section{NUMERICAL PARAMETERS}

\subsection{Computational Domain}

To decrease the computational costs of the simulations, only a periodic portion of the fan stage is simulated. Since the rotor-stator interface condition used for the LES simulation requires to have domains of the same angular extent in the rotor and stator parts, the number of outlet guide vanes has been modified from 54 to 55 allowing a $2 \pi / 11$ angular periodicity in both domains. This geometrical transformation has been performed adjusting the vane aspect ratios to maintain the same solidity and stay as close as possible to the experimental operating point (see Tab. 1). Using the OPTIBRUI platform for the analytical prediction of axial fan noise $[17,14]$, it was verified that similar broadband noise levels should be expected as similar ranges of cut-on modes are found for both configurations. The computational domain shown in Fig. 2 not only contains the fan stage but also the nacelle and outside flow. This avoids defining an inlet condition inside of the turbofan which may affect the acoustic propagation in the nacelle and allows the flow to establish itself from the free flow and the fan rotational speed like in the experimental wind tunnel. Downstream the exiting jet and the mixing layer will also be accounted for in the simulation which also prevents from modifying the acoustic transmission at the end of the nacelle.

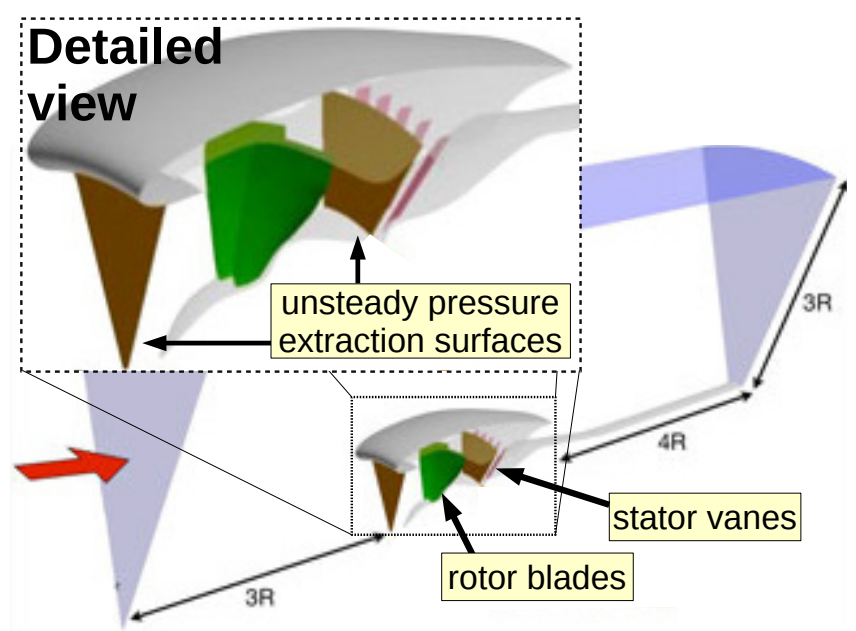

Figure 2. Isometric view of the computational domain.

The domain is divided in two parts: the stator domain is composed of the outside region and the vane row, while the rotor domain is limited to the rotor row and the rotor wake development region. The interfaces between the two parts are shown by the orange surfaces in Fig. 2. 


\subsection{Unstructured Mesh}

The unstructured mesh used in the present study for both RANS and LES simulations is shown along a blade-to-blade view at mid-channel height in Fig. 3. The spatial discretization around the blades and in the wakes of the blades has been a prime focus : 8 layers of prisms have been imposed on the blades and vane skin in order to have a good discretization of the near-wall region. The tip region is discretized with 8 layers of prisms and an average of 7 tetrahedral elements to reach the casing (about 15 overall cells).

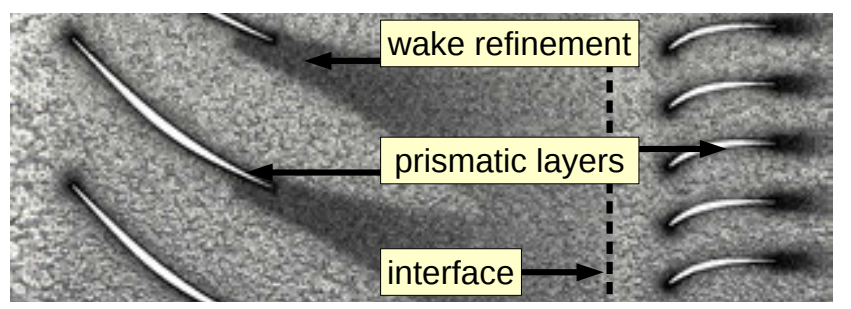

(a) Rotor and stator full blade-to-blade view.

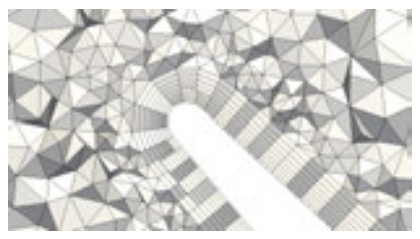

(b) Rotor leading edge.

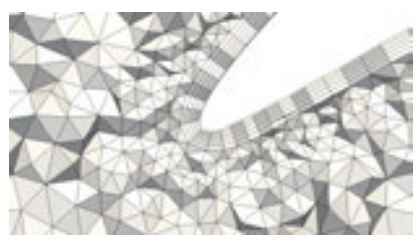

(d) Stator leading edge.

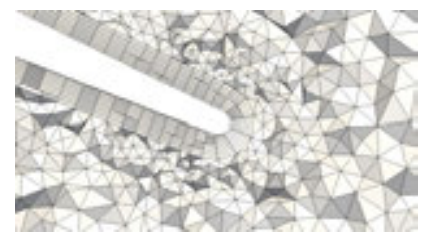

(c) Rotor trailing edge.

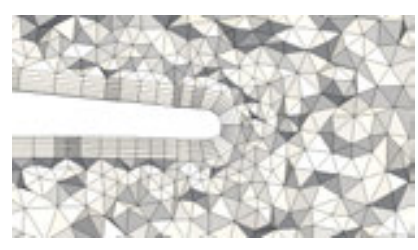

(e) Stator trailing edge.
Figure 3. Grid in a blade-to-blade cut at mid-channel height.

The cell size in the rotor wake is progressively increased until reaching the rotor-stator interface where a constant mesh size of $1.5 \mathrm{~mm}$ is used. Upstream of the rotor blades the mesh is uniform and coarsen up to a mesh size of $3.5 \mathrm{~mm}$ at the entrance of the nacelle. For the outside domain, the nacelle nearby region and the mixing layers are refined. However, the grid is still relatively coarse compared to the inside, the goal being to obtain the mean effect of the mixing layer and not necessary to resolve the turbulent structures precisely. The resulting mesh is composed of 75 million cells ( 15 million nodes) of which over $95 \%$ are used to discretize the inside domain. The domain cut-off frequency is estimated to be about $10 \mathrm{kHz}$ at the inlet interface (upstream of the rotor) and $20 \mathrm{kHz} 1$ stator chord downstream of the stator. The minimum cut-off frequency at the blade skin (used for far-field propagation) is about $35 \mathrm{kHz}$ and $30 \mathrm{kHz}$ for the rotor and stator respectively.

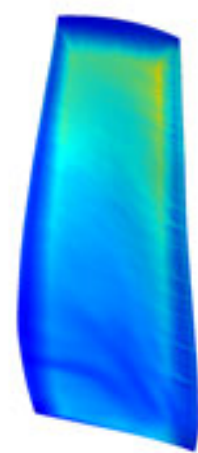

(a)

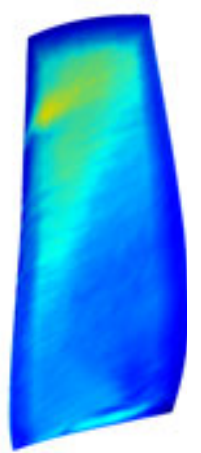

(b)

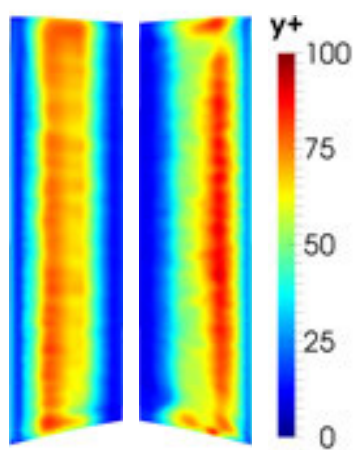

(c)

(d)
Figure 4. Dimensionless wall-normal distance $y^{+}$of the first cell at the wall computed from an average LES. (a) Rotor pressure side. (b) Rotor suction side. (c) Stator pressure side. (d) Stator suction side.

\subsection{Reynolds Averaged Navier-Stokes Simu- lation}

A RANS simulation has been first achieved using the ANSYS CFX v15.0 solver to initialize the LES. The same unstructured mesh as the one used in the LES is used to avoid interpolation errors. A mixing plane condition is used for the rotor-stator interface: only azimuthally averaged fluxes are exchanged. The results presented in this paper were obtained using a $k-\omega$ SST turbulence model with an automatic near-wall treatment that switches from wall-functions to a low-Reynolds near wall formulation as the mesh is refined. After a good initial decay of all residuals for the L2 norm some oscillations are observed which prevent further convergence. They are caused by a leading-edge recirculation bubble that yields a large oscillating flow separation close to the tip of rotor blade (see Fig. 11 in Leonard et al. [14]). However, the oscillations were visible only locally and the simulation reached a steady state in the rest of the domain.

\section{Large Eddy Simulation}

The TurboAVBP code developed by CERFACS and IFPEN [18] has been used for the compressible LES. Two instances of the massively parallel LES solver AVBP are run simultaneously: one for the rotor domain and one for stator. They are coupled by the parallel code coupler OpenPALM [19] which interpolates and exchanges the residuals at each iteration over overlapping grids [20].

The numerical scheme for the present LES is an explicit Lax-Wendroff second order scheme both in space and time. The time step for the simulation has been set to approximately $2.5 \times 10^{-5} \mathrm{~ms}$ to have 14,000 iterations per blade passage. The sub-grid scale model used is the Wall-Adapting Local Eddyviscosity model (WALE) that provides the proper turbulence decay toward the walls. As for the turbine cases [9], a log-law model (which switches to a linear model for $y^{+}<11.445$ ) is applied on all walls to be consistent with the above $y^{+}$ values and to ensure a physical friction on the walls. The 


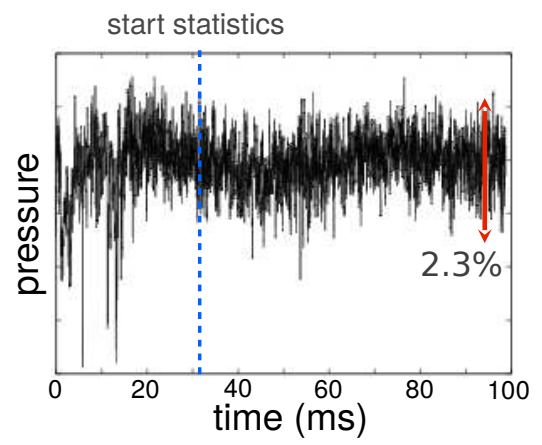

(a) Upstream of the rotor

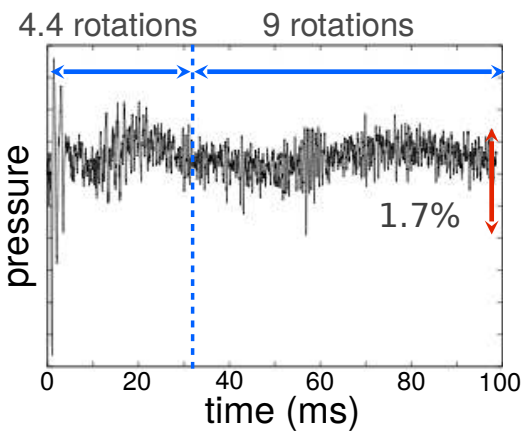

(b) Interstage

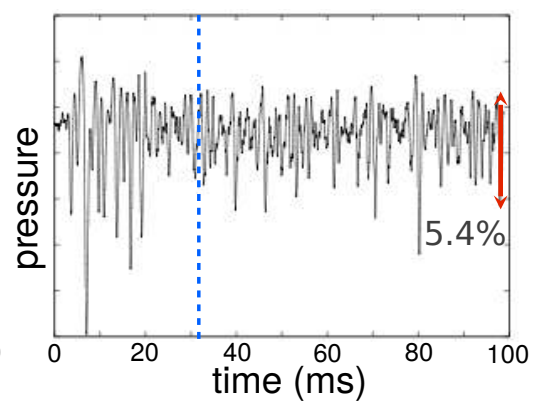

(c) Downstream of the stator

Figure 5. Pressure probes signal at three different locations inside of the turbofan with the end of the transient time highlighted by the vertical dashed line.

dimensionless wall-normal distance $y^{+}$of the first cell at the wall computed from an average solution from the LES is presented in Fig. 4. Thanks to the prisms layers, the $y^{+}$ is under 50 on the whole blade skin, whereas it is coarser on the hub and casing surfaces where prisms have not been used. At the inlet and outlet, Navier-Stokes characteristic non-reflective boundary conditions are used combined with a sponge layer to avoid spurious reflections from vortical fluctuations [21].

The end of the transient period and the beginning of the statistics recording has been chosen according not only to the stabilization of the operating point but also to a method based on local quantities from Mockett et al. for statistical error estimation of finite time signals [22]. To assess this local convergence, several pressure probes have been used in various regions to ensure that the turbulence actually reached a statistically converged regime everywhere. Three convergence plots of those probes are shown in Fig. 5 .

After a transient regime of about 4 rotation times, statistics have been extracted from the simulation during approximately 9 rotations (about $70 \mathrm{~ms}$ or 18 blade passages). The statistical error estimated on the mean and the standard deviation are around $0.2 \%$ and $3.0 \%$ respectively in the fan stage and around $0.2 \%$ and $6.0 \%$ in the jet mixing layer. Overall the convergence of the statistics is very good on both the mean and the Root Mean Square (RMS) of the flow variables. For acoustic post-processing, wall-pressure fluctuations have also been extracted during that time every 20 time steps of the simulation (which corresponds approximately to a 2,000 $\mathrm{kHz}$ sampling rate) on the rotor and stator blades.

The full simulation, including the transient regime, took 15 days on 3000 CPUs on the supercomputer Mammoth-MP2 from Universite de Sherbrooke (1500 CPUs for the static domain, and 1500 CPUs for the rotor domain).

\section{AERODYNAMIC RESULTS}

\subsection{Mean Flow}

The mass-flow rate and total pressure ratio computed from the two simulations are compared in Tab. 1 with measure- ments obtained by Hughes et al. [11]. The overall agreement is quite satisfactory. Similar RANS results were obtained by the various participants of the benchmark with different codes and turbulence models. As expected, predicted performance is different because the simulated tip clearance corresponds to the sideline "hot" geometry with maximum loading that does not account for the same blade deformation in rotation. This also confirms that the slight geometry modifications performed on the stator vane count have a minor impact on the fan stage operating conditions.

Iso-contours of Mach number are presented on bladeto-blade surfaces at three channel heights in Fig. 6. Flow is moving from left to right. Instantaneous LES fields are shown in the left column. The mean fields for the LES and the RANS simulations are seen in the center and right columns respectively. The instantaneous LES snapshots clearly illustrate the turbulent nature of the flow in the stage. At all heights, a quasi laminar flow is seen on the pressure side of the rotor blade and a turbulent boundary layer on its suction side, becoming thicker with increasing radius (increased relative speeds). This yields turbulent rotor wakes convected toward the stator vanes. As was found in the $\mathrm{CME}_{2}$ compressor simulations with three different codes $[7,23,24]$, the wake impingement does not trigger the transition to turbulence at the stator leading edge (too low Reynolds number), which rather occurs at vane mid-chord. Moreover from the instantaneous snapshots at $50 \%$ channel height, an intermittent flow detachment at the leading edge of the rotor is seen to shed vortices that graze along the blade suction sides. A complex turbulent flow behavior is seen at $97 \%$ channel height caused by the tip gap vortices with some intermittent flow separation on one stator-vane pressure side. Note that similar flow behavior has been reported by Casalino et al. using a hybrid Lattice-Boltzmann/Very Large Eddy Simulation (LBM/VLES) model of the baseline SDT configuration [25]. Yet, in this hybrid model a tripping device needed to be introduced close to the blade leading edge over the whole span to trigger transition to turbulence and yield the proper turbulent levels in the wake. No tip gap caused by the voxel resolution could also be captured in the coarsest simulations. 
Large Eddy Simulation of a Scale-model Turbofan for Fan Noise Source Diagnostic - 5/11

\begin{tabular}{|c|c|c|c|}
\hline & Mass-flow rate $(\mathrm{kg} / \mathrm{s})$ & Total pressure ratio & Mean axial speed $(\mathrm{m} / \mathrm{s})$ \\
\hline Experiments[11] & 26.44 & 1.159 & 112.42 \\
\hline RANS & $26.14 \quad(-1.12 \%)$ & $1.160 \quad(+0.09 \%)$ & 107.01 \\
\hline LES & $25.77 \quad(-2.52 \%)$ & $1.162(+0.25 \%)$ & 105.40 \\
\hline
\end{tabular}

Table 1. Fan OGV performances for the baseline configuration at $61.7 \%$ rotor speed (first two columns) and mean axial velocity $\mathrm{V}_{\text {ref }}$ at mid-distance between the rotor and the stator (third column).
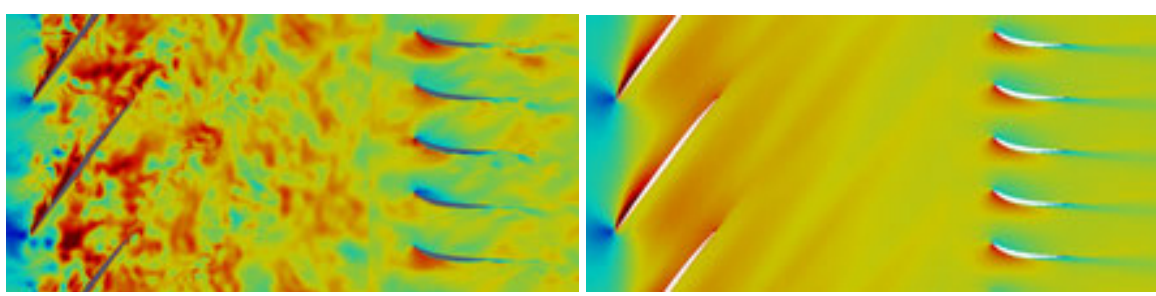

(a) $97 \%$ channel height.
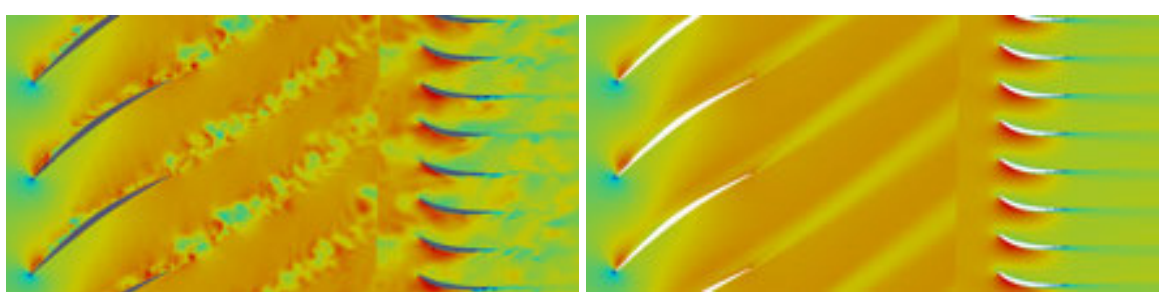

(b) $50 \%$ channel height.
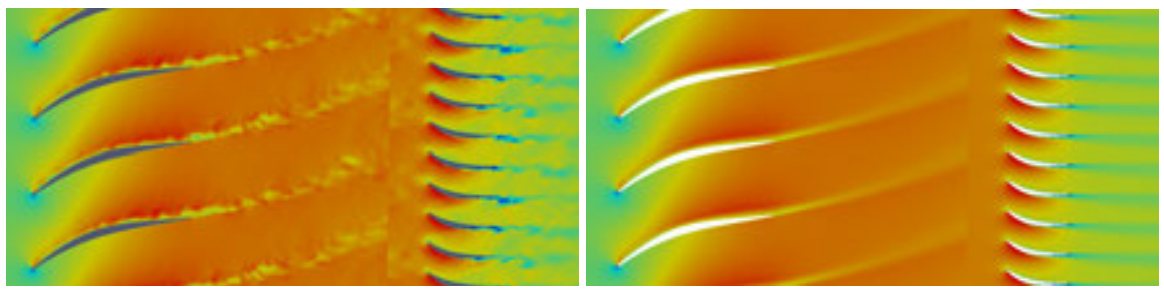

(c) $25 \%$ channel height.
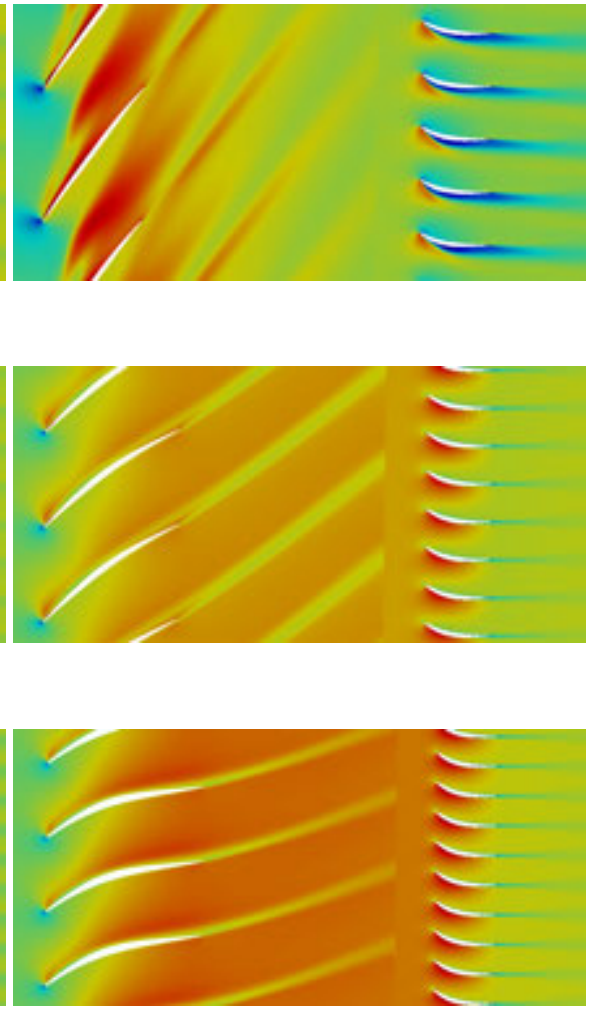

Figure 6. Mach number contours on developed blade-to-blade surfaces. Left: LES instantaneous field. Center: LES averaged field. Right: RANS field.

The mean flow described by both numerical approaches is very similar at midspan. Nevertheless, the LES boundary layers are thinner on the rotor blades (no recirculation bubble as in the RANS) yielding slightly thinner rotor wakes with less velocity deficits than in the RANS results. This effect is emphasized at the hub where the wakes are clearly thinner in the LES than in the RANS simulation. For both spanwise locations, the mean flow in the stator domain is very close in the RANS simulation to the one found in LES in spite of the very different approach used for the rotor-stator interface. Close to the tip, however, the mean flow is significantly different in the two simulations. On the one hand, the RANS calculation predicts a large flow detachment on the rotor blade leading edge which interacts strongly with the tip leakage flow. On the other hand, the deviation induced by the rotor tip flow has an effect on the stator flow resulting in important flow separations at the tip of the outlet guide vanes that do not appear in the LES, which is again quite similar to what was found in the CME2 compressor case [7, 23, 24]. It should be emphasized that such flow separations predicted by the RANS simulations were neither observed experimentally nor in the tripped LBM/VLES case.

Figure 7 presents the field of the normalized component of axial velocity in an axial cut at mid-distance between the rotor trailing edge and the stator leading edge where hotwire (HW) measurements have been performed by Podboy et al. [16]. The values are normalized by the respective (experimental, RANS or LES) mean axial velocity at the axial plane of interest in order to take into account deviations from the experimental operational point given in Tab. 1. The twisting effect on the rotor wake in the interstage by the differential transport of the wake vorticity by the mean swirl is well captured by all simulations. On the upper part of the channel, the radial oscillations (S-shape) observed in the RANS wake 


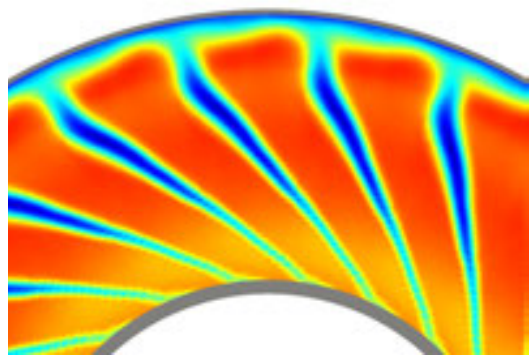

(a) hot-wire

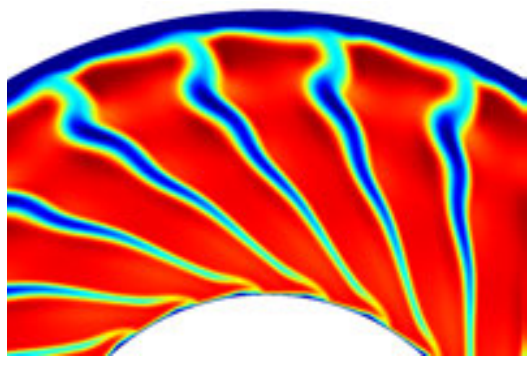

(b) RANS

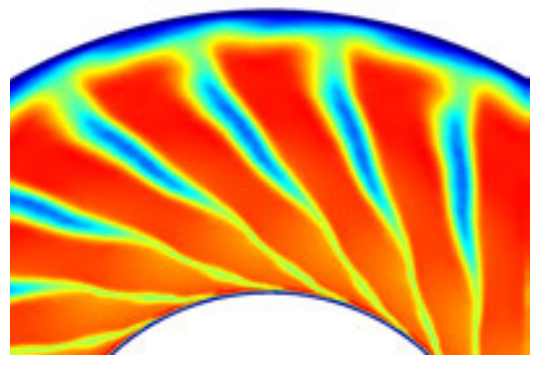

(c) LES

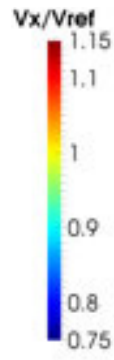

Figure 7. Normalized axial mean velocity flow fields on an axial plane at mid-distance between rotor trailing edge and stator leading edge.

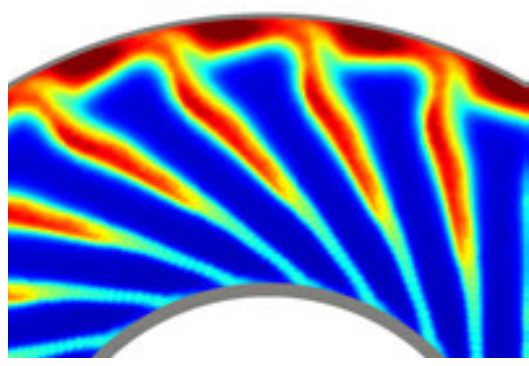

(a) Hot wire: axial

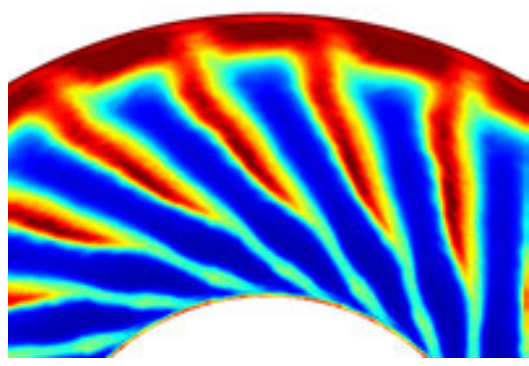

(d) LES: axial

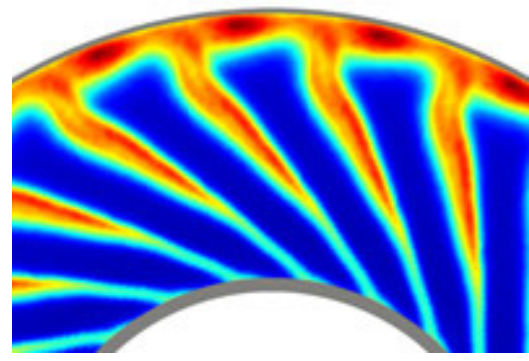

(b) Hot wire: radial

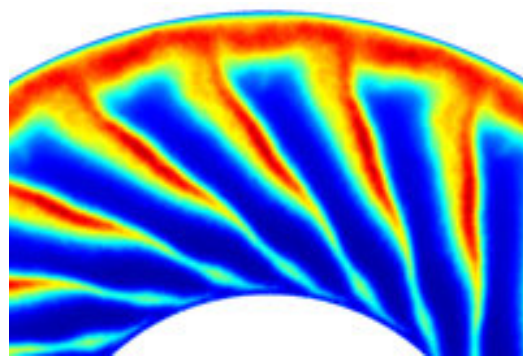

(e) LES: radial

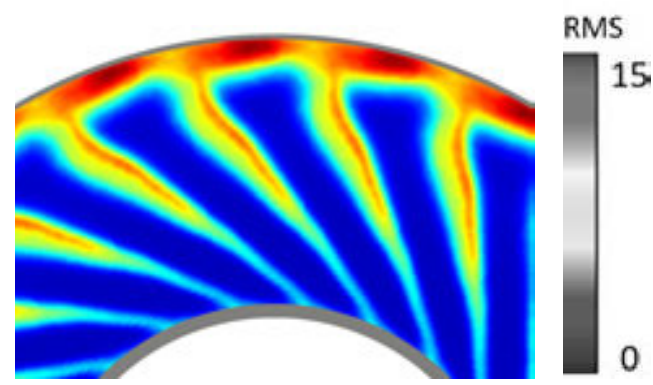

(c) Hot wire: tangential

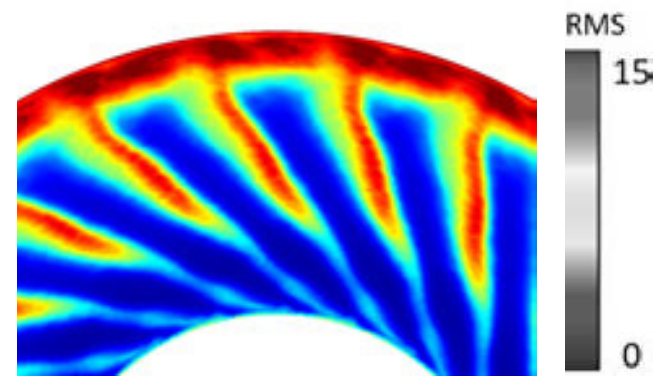

(f) LES: tangential

Figure 8. Three RMS components of the velocity on an axial plane at mid-distance between rotor trailing edge and stator leading edge. Top: hot-wire measurements, bottom: LES.

profile (seen in all benchmark RANS simulations) are the consequence of the tip flow detachment from the leading-edge and is not present in the LES. Both in the LES and RANS the top $10 \%$ of the channel are strongly affected by the tip leakage flow and strong axial velocity deficit are identified caused by the resulting flow blockage. This is hardly seen in the HW measurements, which may still suggest an insufficient mesh resolution in the tip clearance or in the casing boundary layer. Yet as mentioned above, the different geometry used in the simulation does not take into account the same blade deformation in rotation and thus, the gap is different than in the experiments. Finally the LES normalized axial velocity outside the wake agrees well with experiments, even though the wake deficit is under-predicted.

The unsteady nature of the LES also allows to obtain statistics of the resolved turbulent velocity fluctuations: the three RMS components of the velocity are presented in Fig. 8 along with the experimental results. The turbulence pattern obtained by LES, for both the wakes and the tip secondary flow, shows a good agreement with the experiments. In fact, not only the turbulence intensity is accurately reproduced but also the distribution on the three components is very similar all across the channel height with hardly any turbulence near the hub and transition to turbulence at mid-span.

Moreover in the wake, the three components have similar levels in most of the channel height, the tangential component being the lowest of the three. Close to the casing, the tip clearance flows cause a noticeable decrease of the radial fluctuations in favor of the axial and tangential components as already shown by Leonard et al. (Fig. 14 in [14]). Similar results have also been reported by Casalino et al. with a LBM/VLES approach (Fig. 7 in [25]). Overall, the turbulence 
seems to be mostly homogeneous in the wake which has been also highlighted in another turbofan stage studied by Bonneau et al. [26] with a ZDES approach.

\subsection{Unsteady Flow and Noise Sources}

One advantage of LES is to give access to detailed information on the turbulence development and can help understand its influence on the noise sources of the turbofan operating at approach condition. The turbulent structures are identified using an iso-surface of $Q$-criterion computed in the rotor reference frame in Fig. 9. $Q$ stands for the second invariant of the velocity-gradient tensor. Each blade side is shown from its leading edge. As already mentioned for the

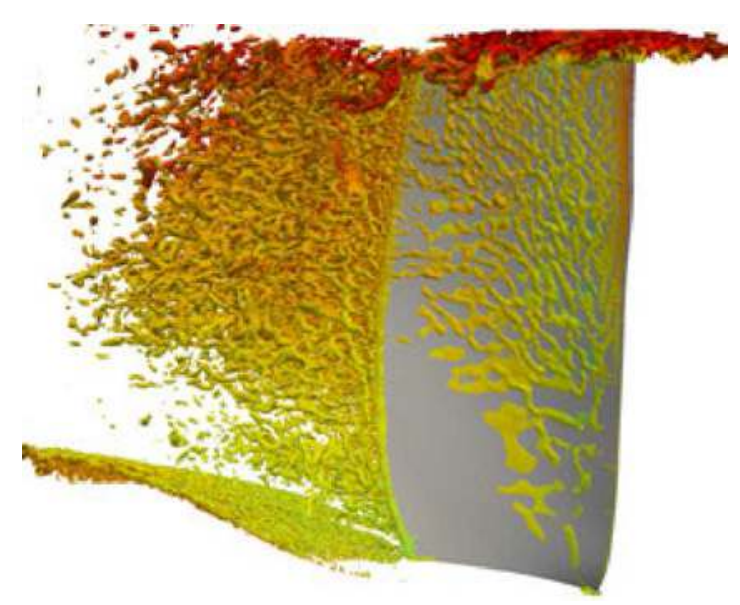

(a) pressure side

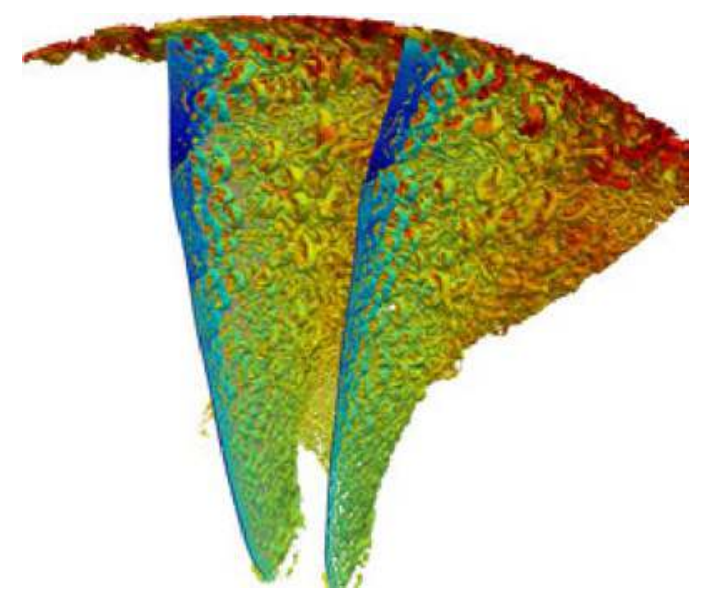

(b) suction side

Figure 9. $Q$ criterion iso-surfaces in the rotor coloured by temperature (leading edge view).

instantaneous Mach number contours, while the boundary layer on the rotor-blade suction side is turbulent starting from the leading edge, the favorable pressure gradient on the pressure side limits the development of the turbulence (quasi laminar along the whole blade span): the boundary layer is initially laminar and some turbulent spots can be localized by the appearance of vertical vortex structures on the upper part of the blade only. On $30 \%$ of the blade span close to the hub, hardly any turbulent structures are visible on the pressure side revealing that the boundary layer stays in a quasi laminar state. The temperature contours coloring the iso- $Q$ further stress that high flow shearing and skin friction typical of turbulent flows only occur on the upper part of both sides of the rotor blades. This yields a very thin wake and low velocity deficit in the lower part of the channel as seen in Fig. 7. The laminar to turbulent boundary layer transition cannot be captured by the RANS approach as it assumes the boundary layer to be turbulent right from the leading edge. This early boundary layer development yields larger wake width in the lower part of the channel in Fig. 7. The tip leakage generates large coherent structures that interact with the pressure side provoking the large velocity deficit in the upper part of the channel.

Dynamic Mode Decompositions (DMD) has been performed on the pressure signals on the rotor blade skin in the rotating reference frame [27]. This signal processing method allows to detect and extract a set of dominant modes in a given complex flow [10]. Figure 10(a) shows the mode associated with the relative vane passing frequency (VPF). Note that the rotor blade is again seen from its leading edge. In the lower part of the blade pressure side where the boundary layer is laminar, a periodic wave going upstream can be seen, representing the acoustic pressure wave generated by wake interaction on the stator vanes traveling upstream. On the suction side a similar pattern can be guessed but it is hidden in the hydrodynamic fluctuations and turbulent vortices.

Further evidence of the above flow and acoustic features is provided by the wall-pressure power spectral densities (PSD) presented in Fig. 10(b). These PSD are computed from the pressure signals of two probes in both the laminar $(15 \%$ of blade span) and turbulent parts (85\% of blade span) of the pressure side of the rotor blade. On the one hand, the spectrum of the probe located in the laminar region has much lower levels with an early and quick roll-off at mid-frequency. It also presents a hump around the stator passing frequency. On the other hand, in the turbulent region the spectrum is broadband with no particular tonal frequency. This acoustic tone is again masked by the turbulent pressure fluctuations.

\section{NOISE PREDICTIONS FROM LES SIMULA- TIONS}

The unsteady pressure recorded on the stator vane skin has been used for far-field noise prediction with two different analogies, the Ffowcs Williams \& Hawkings' (FWH) analogy in free field, and the extension of Goldstein's analogy to annular ducts [28]. The FWH analogy allows to directly propagate the stator wall-pressure fluctuations to the far-field without any duct reflections in order to then compute the acoustic power. Indeed from the resulting acoustic pressure $p^{\prime}(\mathbf{x}, t)$ a surface integration over an upstream half-sphere $S_{-}$and a downstream half-sphere $S_{+}$is performed to evaluate the intake and exhaust power:

$$
S_{p p}^{ \pm}(\omega)=\int_{S_{-} \cup S_{+}} \lim _{T \rightarrow+\infty} \frac{\pi}{T} \mathcal{E}\left(\frac{\left|\widehat{p}^{\prime}(\omega)\right|^{2}}{\rho_{0} c_{0}}\right) d S,
$$




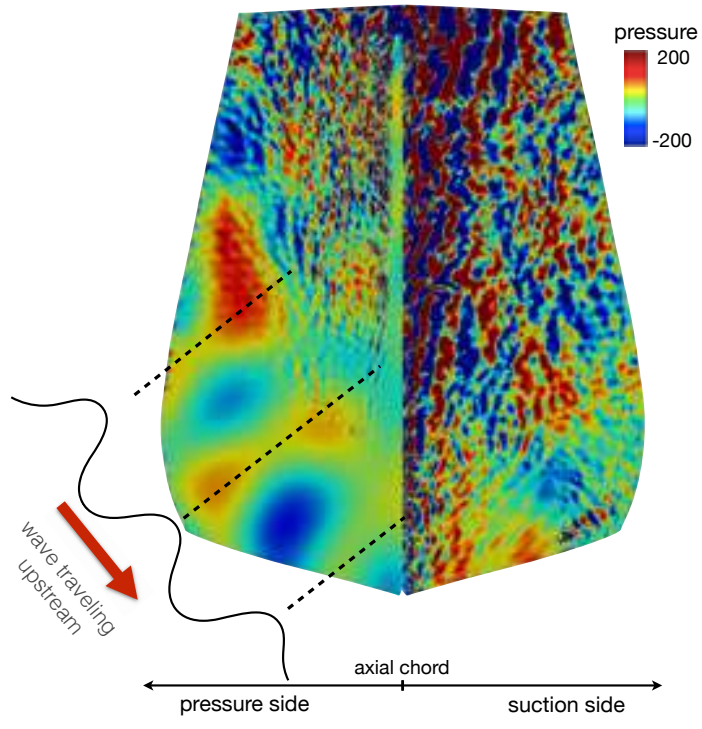

(a) Relative VPF extracted via the DMD on the skin pressure.

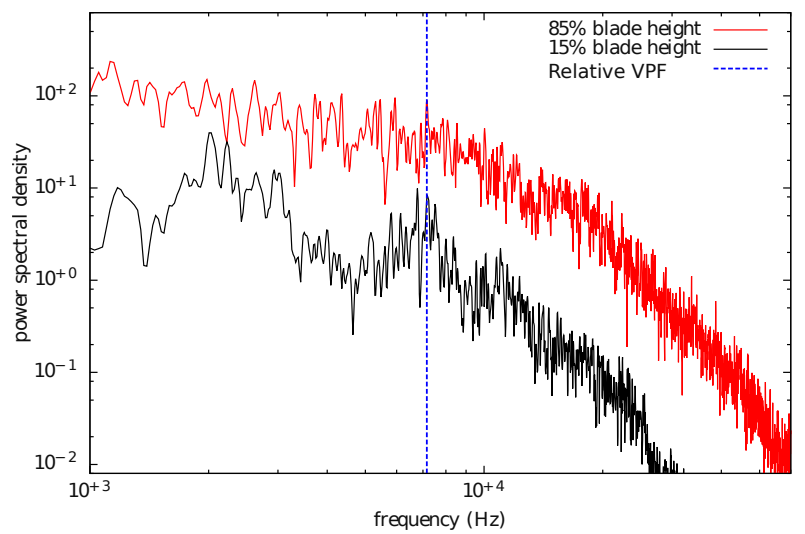

(b) Power spectral density of the pressure signals of two probes at mid chord at two different heights.

Figure 10. DMD analysis on the rotor-blade skin where the relative $\mathrm{BPF}$ refers to the the relative vane-passing frequency for an observer embedded on a moving blade.

where $\mathcal{E}$ represents the expected value (from probability theory) computed from a Welch periodogram. In this work, only the loading noise has been considered as only the pressure fluctuations on the stator vanes are considered. The formulation used in the in-house code SherFWH is reproduced here for completeness. The far-field acoustic pressure at an observer location $\mathrm{x}$ reads

$$
\begin{gathered}
p^{\prime}(\mathbf{x}, t)=\frac{1}{4 \pi c_{0}} \int_{f=0}\left[\frac{\dot{L}_{r}}{r\left(1-M_{r}\right)^{2}}\right]_{\tau} d S \\
+\frac{1}{4 \pi} \int_{f=0}\left[\frac{L_{r}-L_{M}}{r^{2}\left(1-M_{r}\right)^{2}}\right]_{\tau} d S \\
+\frac{1}{4 \pi c_{0}} \int_{f=0}\left[\frac{L_{r}\left(r \dot{M}_{r}+c_{0}\left(M_{r}-M^{2}\right)\right)}{r^{2}\left(1-M_{r}\right)^{3}}\right]_{\tau} d S,
\end{gathered}
$$

where

$$
L_{r}=L_{i} \hat{r}_{i}, \quad \dot{L}_{r}=\dot{L}_{i} \hat{r}_{i}, \quad L_{M}=L_{i} M_{i}, \quad M_{r}=M_{i} \hat{r}_{i}
$$

and dots on quantities denote time derivative with respect to the source time $\tau . L_{i}$ is the loading term and $M_{i}$ is the Mach number of the surface $f=0$ (here the vane surface), $r=|\mathbf{x}-\mathbf{y}|$ where $\mathbf{y}$ is the source position, $\hat{r}_{i}=\left(x_{i}-y_{i}\right) / r$ and $c_{0}$ is the speed of sound in quiescent medium and $t$ the time at the observer. Moreover, the subscript $i$ denotes each coordinate direction and the bracket $[\ldots]_{\tau}$ refers to the evaluation at the retarded time $\tau$.

In Goldstein's analogy extended to annular ducts, the acoustic power $S_{p p}^{ \pm}(\omega)$ at the angular frequency $\omega$ upstream $(-)$ and downstream $(+)$ is computed according to the expression (5a) from [29]:

$$
S_{p p}^{ \pm}(\omega)=\sum_{m \in \mathbb{Z}} \sum_{\mu \in \mathbb{N}} F_{m, \mu}^{ \pm} \lim _{T \rightarrow+\infty} \frac{\pi}{T} \mathcal{E}\left(\left|\mathcal{P}_{m, \mu}^{ \pm}(\omega)\right|^{2}\right)
$$

where $m$ and $\mu$ are the azimuthal and radial orders of an acoustic duct mode respectively. The mode amplitude $F_{m, \mu}^{ \pm}$ links the fluid impedance $\rho_{0} c_{0}$ with $\Gamma_{m, \mu}$, the squared norm of the duct eigenfunction $E_{m, \mu}$ as

$$
F_{m, \mu}^{ \pm}(\omega)=\frac{\Gamma_{m, \mu}}{\rho_{0} c_{0}} G_{m, \mu}^{ \pm}(\omega)
$$

with

$$
G_{m, \mu}^{ \pm}(\omega)=\frac{\kappa_{m, \mu}(\omega) k_{0}(\omega)}{\left|k_{0}(\omega)-k_{m, \mu}^{ \pm}(\omega) M_{x_{d}}\right|^{2}},
$$

where $\kappa_{m, \mu}(\omega)$ is the cut-off wavenumber, $k_{0}(\omega)$ the acoustic wavenumber, $k_{m, \mu}^{ \pm}(\omega)$ the axial wavenumber of the duct mode $(m, \mu)$ in the duct reference frame, and $M_{x_{d}}$ the duct axial Mach number. The latter are defined as

$$
\begin{aligned}
& \kappa_{m, \mu}(\omega)=k_{0}^{2}(\omega)-\beta_{x_{d}}^{2} \chi_{m, \mu}, \quad k_{0}(\omega)=\frac{\omega}{c_{0}}, \\
& k_{m, \mu}^{ \pm}(\omega)=\frac{-k_{0}(\omega) M_{x_{d}} \mp \kappa_{m, \mu}}{\beta_{x_{d}}^{2}}, M_{x_{d}}=\frac{U_{x_{d}}}{c_{0}},
\end{aligned}
$$

where the compressibility factor $\beta_{x_{d}}^{2}=\sqrt{1-M_{x_{d}}}, U_{x_{d}}$ is the axial mean velocity component and $\chi_{m, \mu}$ is the eigenvalue of the mode $(m, \mu)$. In Eq. (4), the pressure amplitude $\mathcal{P}_{m, \mu}^{ \pm}$of the duct mode $(m, \mu)$ can be derived from Eq. (9) from [29] as

$$
\begin{aligned}
\mathcal{P}_{m, \mu}^{ \pm}(\omega) & =-\iint_{\bigcup_{j} S_{j, I}} \frac{\widehat{p}_{w, j}\left(\mathbf{x}_{I 0}, \omega\right)}{2 \mathbf{i} \kappa_{m, \mu}(\omega) \Gamma_{m, \mu}} d \mathrm{~S}_{j, \mathbf{x}_{I 0}} \\
& \cdot \nabla_{I 0}\left(E_{m, \mu}\left(r_{0}\right) e^{-\mathbf{i} m \theta_{I 0}} e^{-\mathbf{i} k_{m, \mu}^{ \pm}(\omega) x_{0}}\right)
\end{aligned}
$$

where the Fourier transform of the pressure jump $\Delta \hat{P}\left(\mathbf{x}_{I 0}, \omega\right)$ has been replaced by the Fourier transform of the elementary force $\mathbf{f}_{j}\left(\mathbf{x}_{I 0}, t_{0}\right)=p_{w, j}\left(\mathbf{x}_{I 0}, t_{0}\right) d \mathbf{S}_{j, \mathbf{x}_{I 0}}$ applied to the surface element of the blade $j$ and the superscript $*$ (conjugate) has been dropped from the duct eigenfunction $E_{m, \mu}$ as it is a real function. In Eq. (8), the subscript $I$ represents 
the reference coordinate system $\mathcal{R}_{I}$, the coordinate vector $\mathbf{x}_{I 0}=\left(r_{I 0}, \theta_{I 0}, x_{I 0}\right)$ constitutes the coordinates of the cellcenters on the surface elements.

Both analogies can be used following two different approaches. The first one is obtained by computing the acoustic power $S_{p p}^{ \pm}(\omega)$ from Eq. (1) or Eq. (4) for FWH and Goldstein's analogy respectively) independently for each blade, and then doing a summation over all of them. This calculation does not take into account any blade-to-blade correlation as the phase is lost when computing the norm in Eqs. (1) and (4). The second approach takes into account blade-to-blade correlations. For the FWH free-field analogy, the acoustic power $S_{p p}^{ \pm}(\omega)$ in Eq. (1) is directly computed from the pressure fluctuations $p^{\prime}$ from Eq. (2) from all the computed blades. Similarly, for Goldstein's analogy, it consists in computing the pressure amplitude $\mathcal{P}_{m, \mu}^{ \pm}$from Eq. (8) for all the blades in the computational domain and then calculating the norm of the expected value. Last, the pressure amplitudes are scaled by the ratio of total vane count and computed vane number. The phase shift of the vanes that are not directly computed is not yet taken into account in this work.

The results from the FWH analogy (noted as FWH Uncorr. and FWH Corr. for the uncorrelated and correlated summation respectively) and for Goldstein's in-duct analogy (noted as Goldstein Uncorr. and Goldstein Corr.) are compared with the measurements [13] in Figs. 11(a) and 11(b). In the experiments, sound power is measured upstream by means of an acoustic screen to hide the downstream contributions. Then this contribution is subtracted from the acoustic power measured without the screen to get the exhaust information. The mock-up was also run without the stator row to measure the contribution from the fan only. The latter is then subtracted from the acoustic power measured with the fan-OGV configuration to isolate the OGV contribution. Unfortunately, the experimental rotor-alone broadband noise levels eventually overtake the fan-OGV noise levels at some frequencies. This is potentially caused by the strong swirling flow at the nacelle exit interacting with the pylon.

The numerical predictions from sources on the stator with both analogies provide globally a good agreement with the experiments both in terms of levels and shape. The largest differences in levels (up to $10 \mathrm{~dB}$ higher than the fan-OGV measurements) are seen at low frequencies. This highlights that additional noise mechanisms may be present in the simulation. Leonard et al. [14] already showed that the rotoralone predictions were overpredicted because of too large coherent structures present in the tip gap region (underresolved region in the LES). Such structures typically yield low-frequency humps observed in low-speed fans [30], which could correspond to the hump above $2 \mathrm{kHz}$ in Figs. 11(a) and $11(\mathrm{~b})$. Moreover, the differences with experiments are higher toward the intake since the reflections on the rotor blades are not taken into account. This effect was shown analytically for the present configuration by Posson and Moreau [31] and numerically by Casalino et al. [25].

The results obtained with Goldstein's analogy extended to annular ducts are closer to the experimental data overall, especially toward the exhaust demonstrating the limitation of using a free-field acoustic propagation for this configuration. The nacelle plays a crucial filtering role on the wave propagation: different duct-modes with distinct energy levels are cut-off yielding the sharper spectral decay at high frequencies seen both experimentally and numerically compared to the free-field case. According to the number of blades and vanes, the BPF and its first harmonics should be cut-off. However, the peak at $2863 \mathrm{~Hz}$ which corresponds to the BPF is visible with both approaches. This is expected because only 5 vanes have been simulated and the Tyler \& Sofrin rules [3] only apply for a full (axisymmetric) configuration. Nonetheless, the peak is reduced by $10 \mathrm{~dB}$ in the second approach when the correlation of the 5 vanes is taken into account. This spurious peak also contributes to the above low-frequency over-prediction which shows the very strong sensitivity of the destructive interference in tone cut-off conditions. Finally the current agreement with experiments at the exhaust is similar to what Casalino et al. obtained with the hybrid LBM/VLES approach (Fig. 12 in [25]), where the LES yields a better high-frequency decay because of the higher resolution of the vane noise sources.

\section{CONCLUSIONS}

The Large Eddy Simulation of the scale-model simplified NASA SDT turbofan has been successfully achieved for the first time at approach condition for the reference stator-vane case. This simulation has been performed on the complete nacelle and fan-OGV experimental set-up with a slightly modified vane count to reduce the computational domain in the azimuthal direction. This simulation was initialized from a preliminary steady RANS simulation of the same setup using a $k-\omega$ SST turbulence model. This configuration is of particular interest since it provides a set of detailed experimental data not only for aerodynamics but also for acoustics. It is then a good test case to evaluate the ability of LES to reproduce the turbulent flow in a fan stage but also to test different acoustic models for noise emission and propagation.

Both RANS and LES results have shown a good overall agreement with experiments on the mean aerodynamic flow properties. However, the RANS simulation presents a leadingedge recirculation bubble at the tip of the rotor blade yielding a downstream S-shape rotor wake, which is not observed in the LES that only presents some intermittent vortex shedding grazing on the suction side. Similarly some flow separation is observed at the tip of the stator vanes in the RANS results, which is neither found in the experiments nor in the LES. Noticeably the LES transitions to turbulence on the rotorblade suction side close to the leading edge because of the sharp local acceleration, which could not be obtained in less resolved hybrid LBM/VLES results without tripping. The comparison of turbulent velocity fluctuations with hot-wire measurements has shown that the LES also gives an accurate representation of turbulence in the wake. Noticeably, the 


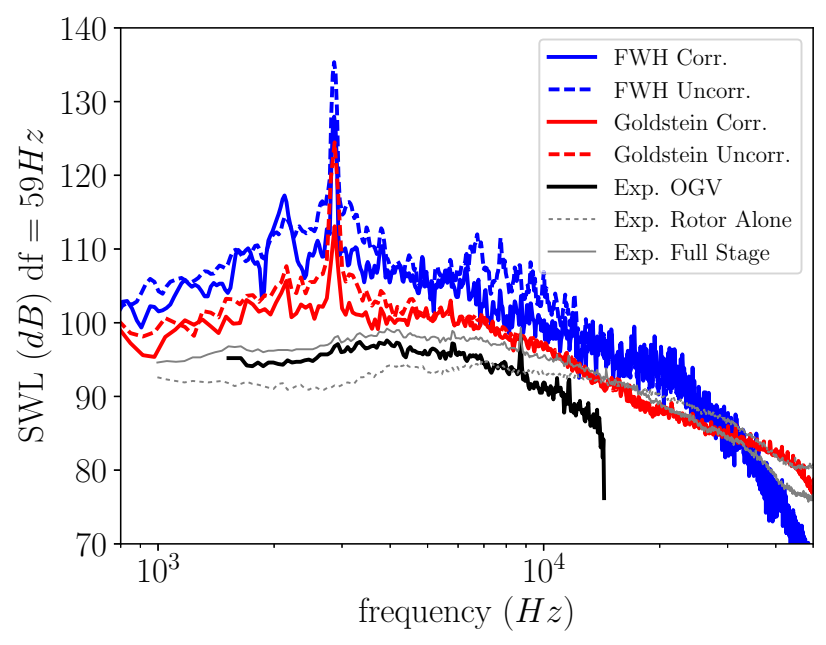

(a) Intake

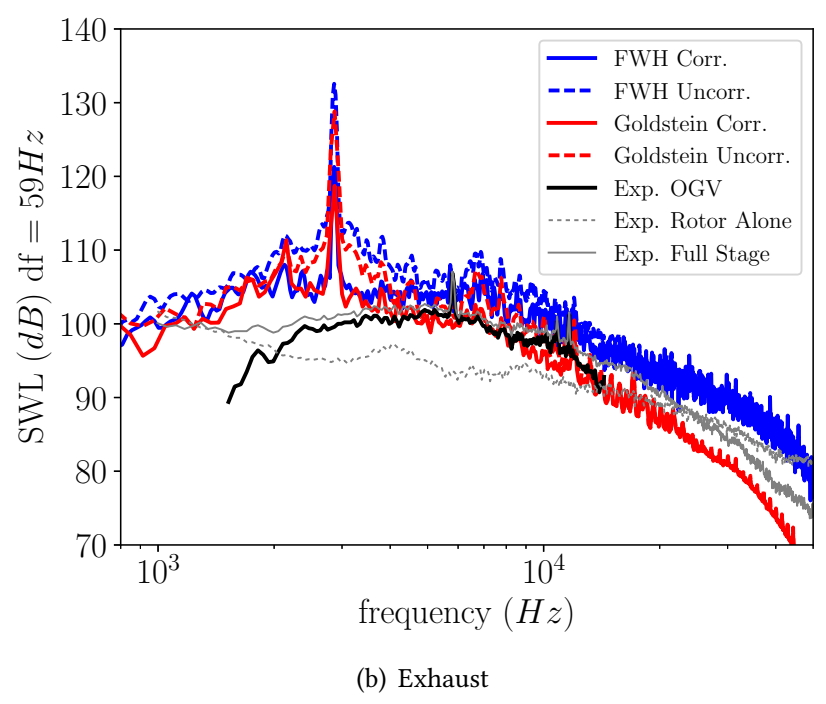

Figure 11. Intake and exhaust sound power levels obtained from the OGV contributions. Rotor only and full stage contributions are added for the experimental results for completeness.

bottom part of the wake is essentially laminar and much thinner in the LES than in the RANS that assumes turbulent flow over the whole passage. The turbulence seems to be mostly isotropic in the wake which confirms the validity of this commonly made hypothesis in most analytical models such as Hanson's or Posson's. Similarly to what was found in low-pressure compressors at similar Reynolds number based on the chord, the transition on the stator vanes is not directly triggered by the rotor wake impingement but rather occurs at mid-chord.

Finally, the LES has the advantage to provide unsteady wall-pressure signals on the stator vanes, which can be used as equivalent noise sources for both Ffowcs Williams \& Hawkings' analogy in free space, and the generalized Goldstein's analogy extended to annular ducts. The sound power level spectra obtained with both analogies present the right trend but over-estimate the noise level at low frequency most likely because the present tip gap is larger than in the experiments and that the tip flow structures are still under-resolved in the present detailed simulation. This is also a consequence of the spurious low-frequency tonal noise caused by the limited number of blades in the present model. The correlated addition of the acoustic power for Goldstein's analogy noticeably reduces the level of this tone. Yet at mid and high frequencies (up to $20 \mathrm{kHz}$ at least), the results from Goldstein's analogy match the experimental OGV spectra well both in terms of levels and shape, much better than the FWH results that overpredict the radiated sound power, which stresses the importance of accounting for the decay of cut-off modes in this frequency range taking place within the duct. An improved agreement over a larger frequency range is also observed compared with the less resolved hybrid LBM/VLES results with tripping.

\section{ACKNOWLEDGMENTS}

Computations were made on the supercomputer Mammoth$\mathrm{MP} 2$ from Universite de Sherbrooke, managed by Calcul Québec and Compute Canada. The operation of this supercomputer is funded by the CFI, NanoQuébec, RMGA and FRQ-NT. The authors would like to thank E. Envia from NASA for fruitful discussions and technical support and for providing the experimental data in the framework of the AIAA benchmark.

\section{REFERENCES}

[1] G. J. J. Ruijgrok. Elements of Aviation Acoustics. Delft University Press, 2004.

[2] Nigel Peake and Anthony B Parry. Modern challenges facing turbomachinery aeroacoustics. Annual Review of Fluid Mechanics, 44:227-248, 2012.

[3] John M Tyler and Thomas G Sofrin. Axial flow compressor noise studies. Technical report, SAE Technical Paper, 1962.

[4] PG Tucker. Computation of unsteady turbomachinery flows: Part 2 - LES and hybrids. Progress in Aerospace Sciences, 47(7):546-569, 2011.

[5] WA McMullan and GJ Page. Towards large eddy simulation of gas turbine compressors. Progress in Aerospace Sciences, 52:30-47, 2012.

[6] Nicolas Gourdain, Frédéric Sicot, Florent Duchaine, and L Gicquel. Large eddy simulation of flows in industrial compressors: a path from 2015 to 2035. Phil. Trans. $R$. Soc. A, 372(2022):20130323, 2014.

[7] J. de Laborderie, S. Moreau, and A. Berry. Compressor Stage Broadband Noise Prediction using a Large-Eddy Simulation and Comparisons with a Cascade Response Model. In 19th AIAA/CEAS Aeroacoustics Conference, AIAA-2013-2042, Berlin, Germany, May 27-29 2013. 
[8] Nicolas Gourdain. Prediction of the unsteady turbulent flow in an axial compressor stage. part 1: Comparison of unsteady rans and les with experiments. Computers \& Fluids, 106:119-129, 2015.

[9] D. Papadogiannis, F. Duchaine, L. Gicquel, G. Wang, and S. Moreau. Effects of Subgrid Scale Modeling on the Deterministic and Stochastic Turbulent Energetic Distribution in Large-Eddy Simulations of a High-Pressure Turbine Stage. Fournal of Turbomachinery, 138(9):091005$091005,2016$.

[10] G. Wang, M. Sanjose, S.e Moreau, D. Papadogiannis, F. Duchaine, and L. Gicquel. Noise mechanisms in a transonic high-pressure turbine stage. International fournal of Aeroacoustics, 15(1-2):144-161, 2016.

[11] C. Hughes, R. Jeracki, R. Woodward, and C. Miller. Fan Noise Source Diagnostic Test - Rotor Alone Aerodynamic Performance Results. In 8th AIAA/CEAS Aeroacoustics Conference \& Exhibit, AIAA-2002-2426, Breckenridge, CO, USA, June 2002. AIAA papers.

[12] G. Podboy, M. Krupar, S. Helland, and C. Hughes. Steady and Unsteady Flow Field Measurements within a NASA 22 inch fan model. In 4oth AIAA Aerospace Sciences Meeting \& Exhibit, AIAA-2002-1033, Reno, NV, USA, January 2002. AIAA papers.

[13] R. Woodward, C. Hughes, R. Jeracki, and C. Miller. Fan Noise Source Diagnostic Test - Far-field Acoustic Results. In 8th AIAA/CEAS Aeroacoustics Conference \& Exhibit, AIAA-2002-2427, Breckenridge, CO, USA, June 2002. AIAA papers.

[14] T. Leonard, M. Sanjosé, S. Moreau, and F. Duchaine. Large Eddy Simulation of a scale-model turbofan for fan noise source diagnostic. In 22nd AIAA/CEAS Aeroacoustics Conference, AIAA Paper 2016-3000. American Institute of Aeronautics and Astronautics, may 2016.

[15] Ed. Envia. Fan Noise Source Diagnostics Test - Vane Unsteady Pressure Results. In 8th AIAA/CEAS Aeroacoustics Conference \& Exhibit, AIAA-2002-2430, Breckenridge, CO, USA, June 2002. AIAA Paper.

[16] G. Podboy, M. Krupar, C. Hughes, and R. Woodward. Fan Noise Source Diagnostic Test - LDV Measured Flow Field Results. In 8th AIAA/CEAS Aeroacoustics Conference \& Exhibit, AIAA-2002-2431, Breckenridge, CO, USA, June 2002. AIAA Paper.

[17] M. Sanjosé, M. Daroukh, W. Magnet, J. De Laborderie, S. Moreau, and A. Mann. Tonal fan noise prediction and validation on the ANCF configuration. Noise Control Engineering fournal, 63(6), 2015.

[18] T. Schönfeld and M. Rudgyard. Steady and Unsteady Flow Simulations Using the Hybrid Flow Solver AVBP. AIAA fournal, 37(11):1378-1385, 1999.

[19] F. Duchaine, S. Jauré, D. Poitou, E. Quémerais, G. Staffelbach, T. Morel, and L. Gicquel. Analysis of high performance conjugate heat transfer with the openpalm coupler. Computational Science \& Discovery, 8(1):015003, 2015.

[20] G. Wang, F. Duchaine, D. Papadogiannis, I. Duran, S. Moreau, and L. Gicquel. An overset grid method for large eddy simulation of turbomachinery stages. Fournal of Computational Physics, 274:333-355, 2014.

[21] T. Poinsot and S. Lele. Boundary conditions for direct simulations of compressible viscous flows. Fournal of Computational Physics, 101(1):104-129, 1992.

[22] C. Mockett, T. Knacke, and T. Thiele. Detection of initial transient and estimation of statistical error in timeresolved turbulent flow data. In Proceedings of 8th International ERCOFTAC Symposium on Engineering Turbulence Modelling and Measurement, 2010.

[23] G. Wang, S. Moreau, F. Duchaine, N. Gourdain, and L. Y. M. Gicquel. Large eddy simulations of the $\mathrm{mt} 1$ high-pressure turbine using turboavbp. In Proceeding of 21st Annual Conference of the CFD Society of Canada, Sherbrooke, Quebec, Canada, May 6-9, 2013.

[24] Nicolas Gourdain. Prediction of the unsteady turbulent flow in an axial compressor stage. part 2: Analysis of unsteady rans and les data. Computers \& Fluids, 106:6778, 2015 .

[25] Damiano Casalino, Andreas Hazir, and Adrien Mann. Turbofan broadband noise prediction using the lattice boltzmann method. In Proceeding of the z2nd AIAA-CEAS Aeroacoustics Conference, 2016.

[26] V. Bonneau, C. Polacsek, L. Castillon, J. Marty, and S. Moreau. Turbofan broadband noise predictions using a $3 \mathrm{D}$ ZDES rotor blade approach. In 22nd AIAA/CEAS Aeroacoustics Conference, AIAA-2016-2950, Lyon, France, May 2016. AIAA Paper.

[27] Peter J Schmid. Dynamic mode decomposition of numerical and experimental data. fournal of fluid mechanics, 656:5-28, 2010.

[28] Marvin E Goldstein. Aeroacoustics. New York, McGrawHill International Book Co., 1976. 305 p., 1, 1976.

[29] H. Posson, S. Moreau, and M. Roger. Broadband noise prediction of fan outlet guide vane using a cascade response function. Fournal of Sound and Vibration, 330:6153-6183, 2011.

[30] S. Moreau and M. Sanjosé. Sub-harmonic broadband humps and tip noise in low-speed ring fans. fournal of the Acoustical Society of America, 139(1):118-127, 2016.

[31] H. Posson and S. Moreau. Effect of Rotor Shielding on Fan-Outlet Guide Vanes Broadband Noise Prediction. AIAA fournal, 51(7):1576-1592, June 2013. 